\title{
Dopamine in the Medial Prefrontal Cortex Controls Genotype-Dependent Effects of Amphetamine on Mesoaccumbens Dopamine Release and Locomotion
}

\author{
Rossella Ventura ${ }^{1,2}$, Antonio Alcaro ${ }^{1,2}$, Simona Cabib ${ }^{1,2}$, Davide Conversi ${ }^{1,2}$, Laura Mandolesi ${ }^{1,2}$ and Stefano \\ Puglisi-Allegra*, ${ }^{*, 2}$ \\ 'Dipartimento di Psicologia, Università 'La Sapienza', Rome, Italy; ${ }^{2}$ Fondazione Santa Lucia IRCCS, Rome, Italy
}

\begin{abstract}
Mice of background DBA/2] are hyporesponsive to the behavioral effects of D-amphetamine in comparison with the widely exploited murine background C57BL/6). In view of the important role of dopamine (DA) release in the nucleus accumbens (NAc) regarding the behavioral effects of psychostimulants, we tested the hypothesis of an inverse relationship between mesocortical and mesoaccumbens DA functioning in the two backgrounds. Systemic D-amphetamine induces a sustained increase in DA release in the medial prefrontal cortex (mpFC) accompanied by a poor increase in the NAc in mice of the low-responsive DBA/2] background, as shown by intracerebral microdialysis in freely moving animals. The opposite occurs in C57BL/6 mice, which show low prefrontal cortical DA outflow accompanied by high accumbal extracellular DA. Moreover, the DBA/2] background showed lower locomotor activity than C57BL/6] mice following D-amphetamine challenge. Selective DA depletion in the mpFC of DBA/2 I mice produced a clear-cut increase in D-amphetamine-induced DA outflow in the NAc as well as locomotor activity that reached levels similar to those observed in C57BL/6) mice. Finally, local infusion of D-amphetamine by reverse microdialysis produced a similar increase in extracellular DA in both the mpFC and the NAc of DBA/2J mice. This finding points to similar transporter-related mechanisms in the two brain areas and supports the hypothesis that low accumbal DA release induced by systemic D-amphetamine in the DBA/2] background is determined by the inhibitory action of prefrontal cortical DA. The present results indicate that genotype-dependent susceptibility to addictive properties of D-amphetamine involves unbalanced DA transmission in the mesocorticolimbic system.
\end{abstract}

Neuropsychopharmacology (2004) 29, 72-80, advance online publication, 10 September 2003; doi: I 0.1038/sj.npp. I 300300

Keywords: dopamine; drugs of abuse; medial prefrontal cortex; nucleus accumbens; mesocorticolimbic system; genotype

\section{INTRODUCTION}

An increasing body of evidence indicates that psychostimulants increase dopamine (DA) transmission in the nucleus accumbens (NAc), the subcortical area that mediates their stimulating/reinforcing effects, through complex neural networks involving a number of brain areas and neurotransmitters (Piazza and Le Moal, 1996; Jackson and Moghaddam, 2001; Porrino and Lyons, 2000). Moreover, DA transmission in subcortical structures, such as the NAc, appears to be modulated by the DA mesocortical system in an inhibitory way (Herve et al, 1981; Deutch et al, 1990; Le Moal and Simon, 1991; Piazza et al, 1991; Thompson and Moss, 1995; Doherty and Gratton, 1996; Karreman and Moghaddam, 1996; Harden

\footnotetext{
* Correspondence: Dr S Puglisi-Allegra, Dipartimento di Psicologia, Università 'La Sapienza', via dei Marsi n. 78, 00185 Rome, Italy, Tel: + 3906 499/7523, Fax: + 3906 49917712,

E-mail: stefano.puglisi-allegra@uniromal.it

Received I5 March 2003; revised 26 July 2003; accepted 29 July 2003 Online publication: 4 August 2003 at http://www.acnp.org/citations/ NPP08040303 I09/default.pdf
}

et al, 1998; Jentsch et al, 1998, Beyer and Steketee, 1999), thus strongly suggesting that the DA response in the accumbens is inversely related to that elicited in the prefrontal cortex.

Mice of the DBA/2J background have been shown to be poorly responsive to the stimulating and the reinforcing effects of D-amphetamine (Cabib et al, 2000) as well as to its enhancing effects on dopamine outflow in the NAc (Zocchi et al, 1998) compared with the widely exploited C57BL/6J background. The C57BL/6J background, commonly used in molecular approaches, has been chosen by the NIH as one of its two standard strains to be evaluated in depth and compared with contrasting inbred strains (The TransNIH Mouse Initiative, http://www.nih.gov/science/Models/ mouse/).

In the light of the aforementioned studies indicating that DA response in the accumbens is inversely related to that elicited in the prefrontal cortex, we hypothesize that this neurobehavioral phenotype depends on high responsiveness of DA transmission in the medial prefrontal cortex $(\mathrm{mpFC})$ of the $\mathrm{DBA} / 2 \mathrm{~J}$ background. 
In particular, a sustained increase in extracellular DA in the mpFC of DBA/2J mice is expected, which would have a strong inhibitory effect on accumbal DA outflow, thus leading to low extracellular DA levels being induced by amphetamine. The opposite is expected in the C57BL/6J background, known to be highly responsive to amphetamine (Cabib et al, 2000), in which low DA outflow in the $\mathrm{mpFC}$ should lead to permissive action on accumbal DA, resulting in high accumbal release.

We also predict that in the DBA/2J background, in the absence of dopaminergic prefrontal cortical influence, amphetamine induces higher DA outflow in the NAc and higher locomotor activity. This can be assessed by evaluating the effects of selective prefrontal dopamine depletion on those produced by systemic amphetamine on accumbal DA and behavior.

Lastly, if low DA outflow in the NAc of DBA/2J mice is due to the inhibitory action of prefrontal DA on accumbal $\mathrm{DA}$, it is conceivable that locally infused amphetamine does produce a similar increase in extracellular DA in both the $\mathrm{NAc}$ and the $\mathrm{mpFC}$, unless dopamine transporter (DAT)related mechanisms lead to differences in DA release in the two brain areas (Moron et al, 2002).

Here, we report the results of experiments aimed at assessing (1) if in DBA/2J mice, systemic amphetamine induces a sustained increase of DA release in the mpFC accompanied by a low increase in the NAc, and if the opposite occurs in mice of the C57/BL/6J background, which is highly responsive to amphetamine; (2) if selective prefrontal DA depletion in the DBA/2J background potentiates the effects of the psychostimulant on accumbal DA and on locomotion; (3) if, unlike systemic administration, intracortical or intra-accumbens infusion of amphetamine in the DBA/2J background produces a similar increase in dopamine release.

\section{MATERIALS AND METHODS}

\section{Animals}

Male mice of the inbred C57BL/6JIco (C57) and DBA/2JIco (DBA) strains (Charles River Italy) were used for these experiments. All mice were purchased at 6 weeks of age. Upon their arrival, animals were housed in groups of four per standard breeding cage $\left(27 \times 21 \times 13.5 \mathrm{~cm}^{3}\right)$ with food and water ad libitum on a $12 / 12 \mathrm{~h}$ dark/light cycle (lights on between 0700 and 1900). Experiments started when animals reached 8 weeks of age and were carried out in a room separated from the colony room between 1400 and 1800. All the mice used were handled and accustomed to the environment where the experiment was to be performed and then randomly assigned to different treatments. Each experimental group comprised six to 11 animals. All mice were housed individually $24 \mathrm{~h}$ before surgery for microdialysis. Naive animals were used for each experiment.

The procedures used in this study were in strict accordance with European legislation (EEC no. 86/609), with Italian national legislation (DL no. 116/92) governing the use of animals for research, and with the guidelines of the National Institutes of Health on the use and care of laboratory animals.

\section{Drugs}

D-Amphetamine sulfate (amphetamine), chloral hydrate, 6-hydroxydopamine (6-OHDA), and desipramine hydrochloride (DMI) were purchased from Sigma (Sigma Aldrich, MI). Amphetamine $(2.5 \mathrm{mg} / \mathrm{kg})$, chloral hydrate $(450 \mathrm{mg} /$ $\mathrm{kg})$, and DMI $(35 \mathrm{mg} / \mathrm{kg})$ were dissolved in saline $(0.9 \%$ $\mathrm{NaCl}$ ) and injected intraperitoneally (i.p.) in a volume of $10 \mathrm{ml} / \mathrm{kg}$. Amphetamine $(1,5,10,100,1000 \mu \mathrm{M})$ was dissolved in artificial CSF for reverse microdialysis experiments. 6-OHDA was dissolved in saline containing Na-metabisulfite $(0.1 \mathrm{M})$.

\section{Microdialysis}

Animals were anesthetized with chloral hydrate, mounted in a stereotaxic frame (David Kopf Instruments, Tujunga, CA) equipped with a mouse adapter and implanted unilaterally with a guide cannula (stainless steel, shaft OD $0.38 \mathrm{~mm}$, Metalant AB, Stockholm, Sweden,) in the mpFC or in the NAc. The length of the guide cannula was $1 \mathrm{~mm}$ for mpFC and $4.5 \mathrm{~mm}$ (C57) or $4.0 \mathrm{~mm}$ (DBA) for NAc. The guide cannula was fixed with epoxy glue, and dental cement was added for further stabilization. The coordinates from bregma (measured according to the atlas of Franklin and Paxinos (1998) and Mouse Brain Atlases, The Mouse Brain Library, www.nervenet.org/mbl/) were as follows: $m p F C$ : $\mathrm{C} 57=+2.5$ AP, $0.6 \mathrm{~L} ; \mathrm{DBA}=+2.0$ AP, $0.6 \mathrm{~L} ; N A c$ : $\mathrm{C} 57=+1.6$ AP, $0.6 \mathrm{l}$; DBA: +1.1 AP, 0.6 L. The probe (dialysis membrane length $2 \mathrm{~mm}$ for $\mathrm{mpFC}$ and $1 \mathrm{~mm}$ for NAc; OD $0.24 \mathrm{~mm}, \mathrm{MAB} 4$ cuprophane microdialysis probe, Metalant $\mathrm{AB}$ ) was introduced $24 \mathrm{~h}$ after implantation of the guide cannula. The probe was then fastened with the locking system provided by Metalant $\mathrm{AB}$. The animals were lightly anesthetized to facilitate manual insertion of the microdialysis probe into the guide cannula. The membranes were tested for in vitro recovery of DA (relative recovery $(\%)=10.9+0.86 ; n=21)$ on the day before use to verify recovery.

The microdialysis probe was connected to a CMA/100 pump (Carnegie Medicine Stockholm, Sweden) through a PE-20 tubing and an ultralow torque dual channel liquid swivel (Model 375/D/22QM, Instech Laboratories, Inc., Plymouth Meeting, PA) to allow free movement. Artificial CSF (147 mM NaCl, $2.2 \mathrm{mM} \mathrm{CaCl}_{2}$ and $4 \mathrm{mM} \mathrm{KCl}$ ) was pumped through the dialysis probe at a constant flow rate of $2 \mu \mathrm{l} / \mathrm{min}$. Experiments were carried out $22-24 \mathrm{~h}$ after probe placement. Each animal was placed in a circular cage provided with microdialysis equipment (Instech Laboratories, Inc.) and with home cage bedding on the floor. Dialysis perfusion was started $1 \mathrm{~h}$ later. Following the start of the dialysis perfusion, mice were left undisturbed for approximately $2 \mathrm{~h}$ before the collection of baseline samples. The dialysate was collected every $20 \mathrm{~min}$ for $180 \mathrm{~min}$. Placements were judged by methylene blue staining. Only data from mice with correctly placed cannula have been reported. Within the $\mathrm{mpFC}$, the probe position mainly included the prelimbic cortex in both strains (Figure 1). Within the NAc, the probe position mainly included the shell subdivision (Figure 2). In total, $20 \mu \mathrm{m}$ of the dialysate samples was analyzed by high-performance liquid chromatography (HPLC). The remaining $20 \mu \mathrm{l}$ were kept for 

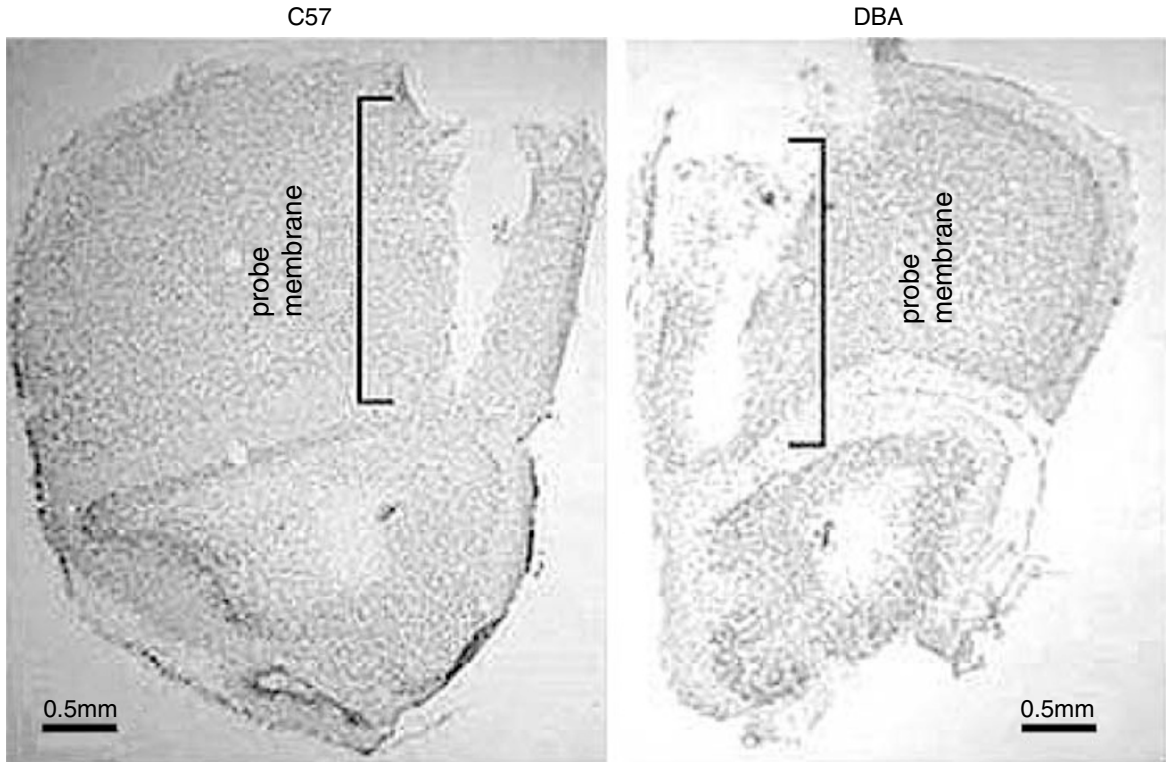

Figure I Location of microdialysis probe in the mpFC of C57BL/6 (C57) and DBA/2J (DBA) mice. Nissl's stained coronal sections of mouse brain hemispheres show the segment of the probe membrane in the two backgrounds.

possible subsequent analysis. Concentrations (pg/20 $\mu \mathrm{l})$ were not corrected for probe recovery. The mean concentration of the three samples collected immediately before treatment (less than $10 \%$ variation) was taken as the basal concentration. The average basal values of dopamine for each group did not differ significantly. Therefore, they have been grouped together here. Basal values $(\mathrm{pg} / 20 \mu \mathrm{l})$ were as follows: $m p F C$ : DA: $\mathrm{C} 57=0.286 \pm 0.013$; $\mathrm{DBA}=0.356 \pm$ 0.006 ; NAc: DA: $\mathrm{C} 57=1.27 \pm 0.14$; DBA $=1.38 \pm 0.23$.

For reverse microdialysis experiments, increasing doses of amphetamine $(1,5,10,100,1000 \mu \mathrm{M})$ were perfused for $60 \mathrm{~min}$ each (three blocks of $20 \mathrm{~min}$ summed over each $60 \mathrm{~min}$ point) (Hedou et al, 2000).

The HPLC system consisted of an Alliance (Waters Corporation, Milford, MA) system and a coulometric detector (ESA Model 5200A Coulochem II) provided with a guard cell (M 5021) and an analytical cell (M 5011). The guard cell was set at $400 \mathrm{mV}$, electrode 1 at $200 \mathrm{mV}$, and electrode 2 at $-250 \mathrm{mV}$. A Nova-Pack C18 column $\left(3.9 \times 150 \mathrm{~mm}^{2}\right.$, Waters $)$ maintained at $33^{\circ} \mathrm{C}$ was used. The flow rate was $1.1 \mathrm{ml} / \mathrm{min}$. The mobile phase was as previously described (Westerink et al, 1998). The detection limit of the assay was $0.1 \mathrm{pg}$.

\section{Visualization of Probe Placement}

Two types of visualization of probe placement were carried out. Visualization in the mpFC was evidentiated by Nissl's staining. In addition, we used DAT immunostaining in order to identify core-shell borders in the NAc of each strain. Indeed, a number of studies have shown differential expression of DAT in the NAc shell and core at the regional as well as subcellular level (Jones et al, 1996; Nirenberg et al, 1997).

Animals were deeply anesthetized with chloral hydrate and transcardially perfused with saline followed by ice-cold
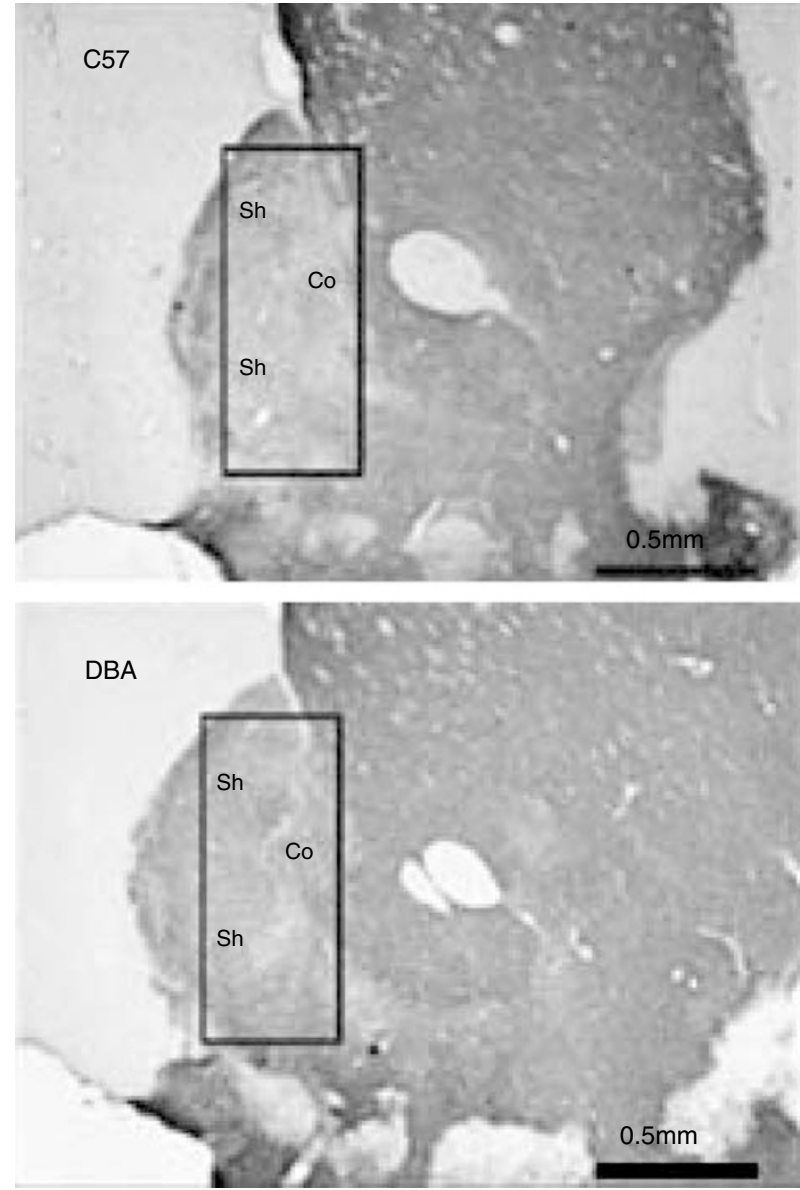

Figure 2 Anatomical location of microdialysis probes in the NAc of C57BL/6J (C57) and DBA/2J (DBA) mice. The inset represents the areas containing the tracks of the microdialysis probes and the range of implantation sites. DAT immunostaining was used to reveal core-shell borders. Sh $=$ NAc shell; Co $=$ NAc core. 
$10 \%$ neutral buffered formalin. Brains were dissected, further fixed, and cryoprotected in $30 \%$ sucrose.

The frontal lobes were dissected, and the extent of the cortical lesion was determined from Nissl-stained $40 \mathrm{~mm}$ frozen sections (Figure 1).

Sections including the NAc ( $30 \mu \mathrm{m}$ sections) were serially collected in $10 \mathrm{mM} \mathrm{pH} 7.4$ phosphate-buffered saline (PBS) and processed for DAT immunostaining (Figure 2). Sections were processed free-floating at room temperature in 24-well culture plates placed on gentle orbital agitation. After a 15 min endogenous peroxidase inactivation step with $0.3 \%$ hydrogen peroxide in PBS, sections were rinsed in PBS, then incubated overnight in a primary antibody (monoclonal rat anti-DAT, Sigma) diluted $1 / 10000$ in PBS with $1 \%$ bovine serum albumin (BSA) and $0.25 \%$ Triton X-100. After rinsing in PBS, sections were incubated for $1.30 \mathrm{~h}$ in secondary antiserum (polyclonal mouse anti-rat, Jackson immunoresearch) diluted $1 / 500$ in PBS with $1 \%$ BSA and $0.25 \%$ Triton X-100. Sections were then thoroughly rinsed and incubated for $1 \mathrm{~h}$ in an avidin-biotin complex (Vector Laboratories) diluted $1 / 500$ in PBS with $0.25 \%$ Triton X-100. After further rinsing, tissue-bound horseradish peroxidase was revealed by incubating sections in cobalt-enhanced diaminobenzidine substrate solution prepared according to the manufacturer's instructions (Sigma). After rinsing in $10 \mathrm{mM}$ pH 8.0 Tris-buffered saline, sections were mounted on superfrost slides, dehydrated with ascending grades of $\mathrm{EtOH}$, cleared in xylene, and coverslipped with Entellan (Merck).Visual examination and digital imaging were performed with a light-transmission microscope equipped with a CCD camera.

\section{Dopamine Depletion}

Anesthesia and surgical set have been described in the previous section.

DBA mice were injected with DMI $(35 \mathrm{mg} / \mathrm{kg}) 30 \mathrm{~min}$ before 6-OHDA microinjection in order to protect noradrenergic neurons. Bilateral injections of 6-OHDA $(1.5 \mu \mathrm{g} /$ $0.1 \mu \mathrm{l} / 2 \mathrm{~min}$ for each side) were made into the mpFC (coordinates: $+2.0 \mathrm{AP} ; \pm 0.6 \mathrm{~L} ;-2.0 \mathrm{~V}$ with respect to bregma) through a stainless-steel cannula $(0.15 \mathrm{~mm} \mathrm{OD}$, UNIMED, Switzerland) connected to a $1 \mu \mathrm{l}$ syringe by a polyethylene tube and driven by a CMA/100 pump. The cannula was left in place for an additional 2 min after the end of the infusion. Sham animals were subjected to the same treatment, but received intracerebral vehicle.

Norepinephrine (NE) and DA tissue levels in the mpFC were assessed as previously described (Ventura et al, 2003) to evaluate the amount and the extent of depletion. The brain was fixed vertically on the freeze plate of a freezing microtome. Punches of both hemispheres were obtained from brain slices (coronal sections) no thicker than $300 \mu \mathrm{m}$. Stainless-steel tubing of $2.3 \mathrm{~mm}$ internal diameter was used. The coordinates were measured according to the atlas of Franklin and Paxinos (1998) and adapted according to the Mouse Brain Atlases (The Mouse Brain Library, www.nervenet.org/mbl/) and to the previous study (Ventura et al, 2001). The punches were stored in liquid nitrogen until the day of analysis.

DA and NE were determined simultaneously, utilizing a reverse phase HPLC procedure coupled with coulochem electrochemical detection. On the day of the analysis, frozen samples were weighed and homogenized in $\mathrm{HC1O}_{4} 0.1 \mathrm{~N}$ containing Na-metabisulfite $6 \mathrm{mM}$ and EDTA $1 \mathrm{mM}$. The homogenates were centrifuged at $10000 \mathrm{rpm}$ for $20 \mathrm{~min}$ at $4{ }^{\circ} \mathrm{C}$. Aliquots of the supernatant were then transferred to the HPLC system.

The HPLC system is described above, the potentials being set at +450 and $+100 \mathrm{mV}$ at the analytical and the conditioning cell, respectively. The columns, a Nova-Pack Phenyl column $\left(3.9 \times 150 \mathrm{~mm}^{2}\right)$, and a Sentry Guard NovaPack precolumn $\left(3.9 \times 20 \mathrm{~mm}^{2}\right)$, were purchased from Waters Assoc. The flow rate was $1 \mathrm{ml} / \mathrm{min}$. The mobile phase consisted of $3 \%$ methanol in $0.1 \mathrm{M}$ Na-phosphate buffer $\mathrm{pH} 3, \mathrm{Na}_{2}$ EDTA $0.1 \mathrm{mM}$, and 1-octane sulfonic acid $\mathrm{Na}$ salt (Aldrich) $0.5 \mathrm{mM}$.

Animals were used for microdialysis or behavioral experiments 7 days after surgery.

\section{Locomotor Activity}

Locomotor activity was assessed in animals other than those tested in microdialysis experiments. They were either naive (C57 and DBA) or had been subjected to DA depletion as described in the previous section (DBA: Sham or DA depleted). Mice from each background (C57 or DBA) or pretreatment (Sham or DA depleted) were challenged with amphetamine or vehicle before testing. The apparatus comprised eight gray opaque Plexiglas chambers divided into two compartments $(20 \times 10)$, with removable floors, placed inside a sound-attenuated room. Individual mice were introduced into each chamber and accustomed to the apparatus for $60 \mathrm{~min}$. Then, all mice were removed and left undisturbed inside their home cages for the following $60 \mathrm{~min}$. Immediately before testing, subjects were weighed and injected (i.p.) with $2.5 \mathrm{mg} / \mathrm{kg}$ of amphetamine or with $10 \mathrm{ml} / \mathrm{kg}$ of vehicle. Then, mice were placed individually in the same cages they had experienced and tested for the following $60 \mathrm{~min}$.

Behavioral data were collected and analyzed by the 'EthoVision' (Noldus, The Netherlands) fully automated video tracking system (Spink et al, 2001). Briefly, a CCD video camera was used to record the experimental system. The signal was then digitized (using a hardware device called a frame grabber) and passed on to the computer's memory. Later on, digital data were analyzed using EthoVision software to measure the number of crossings between compartments.

\section{Statistics}

Microdialysis data were analyzed for each brain area by repeated-measures ANOVA with two between factors (treatment, two levels: saline, amphetamine; strain, two levels: C57, DBA, or pretreatment, two levels: Sham, DA depleted), and one within factor (time, seven levels: 0, 20, $40,60,80,100,120$ min from drug injection).

Reverse microdialysis was analyzed by repeated-measures ANOVA with one within factor (doses, six levels: basal, 1, 5, $10,100,1000 \mu \mathrm{M})$.

The locomotor activity was analyzed by two-way ANOVA, the factors being: strain (two levels: C57, DBA) or pretreatment (two levels: Sham, DA depleted) and treatment 
(two levels: saline, amphetamine). In the case of significant interactions, post hoc comparisons were performed by a Duncan test. The Student's $t$-test (two-tailed) was also used.

\section{RESULTS}

Effects of Systemic Amphetamine on Extracellular DA in the mpFC and the NAc and on Locomotion in C57 and DBA Mice

The effects of amphetamine on DA release in the mpFC are shown in Figure 3. Statistical analyses revealed a significant strain $\times$ treatment $\times$ time interaction $\quad(\mathrm{F}(1,180)=12.8$; $p<0.0005)$. A simple effect analysis showed a significant effect of time only for amphetamine and a significant difference between saline and amphetamine. Moreover, significant differences between C57 and DBA mice challenged with the psychostimulant were evident. Amphetamine produced a time-dependent increase in DA outflow in the mpFC of both strains, but DBA mice showed dramatically higher levels than the C57 background throughout (20-120 min). Extracellular DA reached $\sim 700 \%$ maximal increase (at $40 \mathrm{~min}$ ) in DBA mice, while $\sim 150 \%$ maximal increase was evident (at $40 \mathrm{~min}$ ) in the C57 background. No significant differences were evident in the basal levels between the two backgrounds.

The effects of amphetamine on DA release in the NAc are shown in Figure 3. Statistical analyses revealed a significant strain $\times$ treatment $\times$ time interaction $(\mathrm{F}(1,144)=5.13$; $p<0.001)$. A simple effect analysis showed a significant effect of time only for amphetamine and a significant difference between saline and amphetamine. Moreover, significant differences between C57 and DBA mice challenged with the psychostimulant were evident. Amphetamine produced a time-dependent increase in DA outflow in the NAc of both strains, but C57 mice showed significantly higher levels from 20 to $60 \mathrm{~min}$. Extracellular DA reached $\sim 150 \%$ maximal increase (at $40 \mathrm{~min}$ ) in DBA mice, while $\sim 350 \%$ maximal increase was evident (at $40 \mathrm{~min}$ ) in the C57 background. No significant differences were evident in basal levels between the two backgrounds.

The effects of amphetamine on locomotor activity in mice of C57 and DBA strains are shown in Figure 4. ANOVA showed significant strain $\times$ treatment interaction $(\mathrm{F}(1,28)=7.26 ; p<0.05)$. Individual between group comparisons showed significant differences between mice of both backgrounds challenged with amphetamine in comparison with mice of the same background challenged with saline. Amphetamine increased locomotor activity in both backgrounds, but mice of DBA background showed significantly lower activity scores than the C57 background.

\section{Effects of Selective DA Depletion in the mpFC on DA Release in the NAc and Locomotion in DBA Mice Challenged with Amphetamine}

Intracortical neurotoxin (6-OHDA) reduced DA levels in the $\mathrm{mpFC}$ by about $90 \%$ in comparison with Sham animals, while nonsignificant changes were evident in the NE content (Table 1). These results show that our experimental procedure allows a selective depletion of DA prefrontal
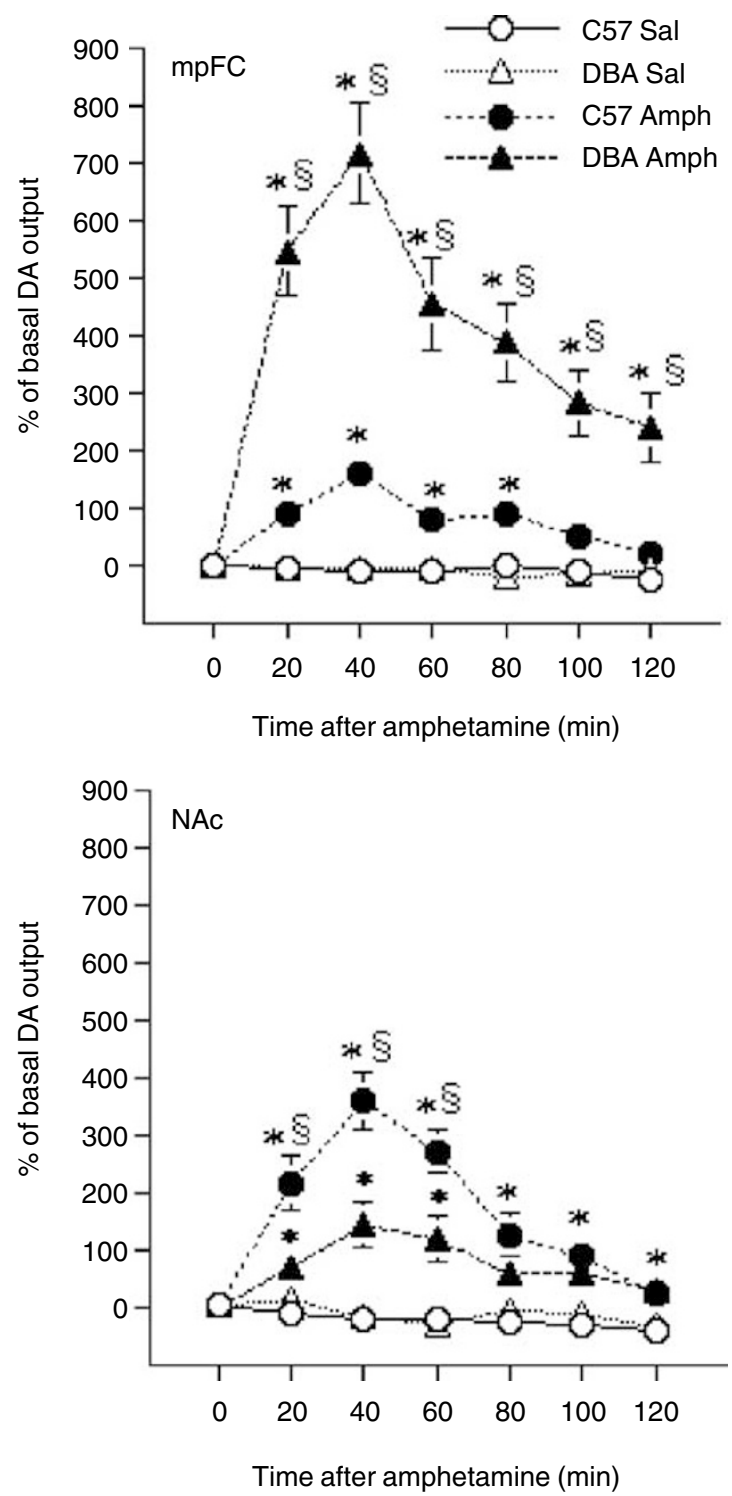

Figure 3 Extracellular dopamine in the $\mathrm{mpFC}$ and $\mathrm{NAc}$ of C57BL/6) (C57) and DBA/2J (DBA) mice ( $n=7-1$ I per group) receiving saline (Sal) or amphetamine ( $2.5 \mathrm{mg} / \mathrm{kg}$, i.p.) (Amph). Results are expressed as percent changes from basal levels. Statistical analyses were carried out on raw data (mean $\pm \mathrm{SE}$ ). $* p<0.005$ compared with saline; $\$ p<0.005$ compared with the other strain.

cortical afferents, and is thus suitable for the purposes of the present study.

The effects of selective DA depletion in the mpFC on DA release in the NAc are shown in Figure 5. Statistical analyses revealed significant pretreatment $\times$ treatment $\times$ time interaction $(\mathrm{F}(1,120)=10.05 ; p<0.0005)$. A simple effect analysis revealed a significant effect of time only for amphetamine and a significant difference between saline and amphetamine. Moreover, significant differences between Sham and DA-depleted groups injected with amphetamine were evident. Amphetamine produced a significant increase in DA release in the NAc of both Sham and DAdepleted groups, but selective prefrontal cortical DA depletion led to a clear-cut potentiation of amphetamineinduced increase in DA outflow. 


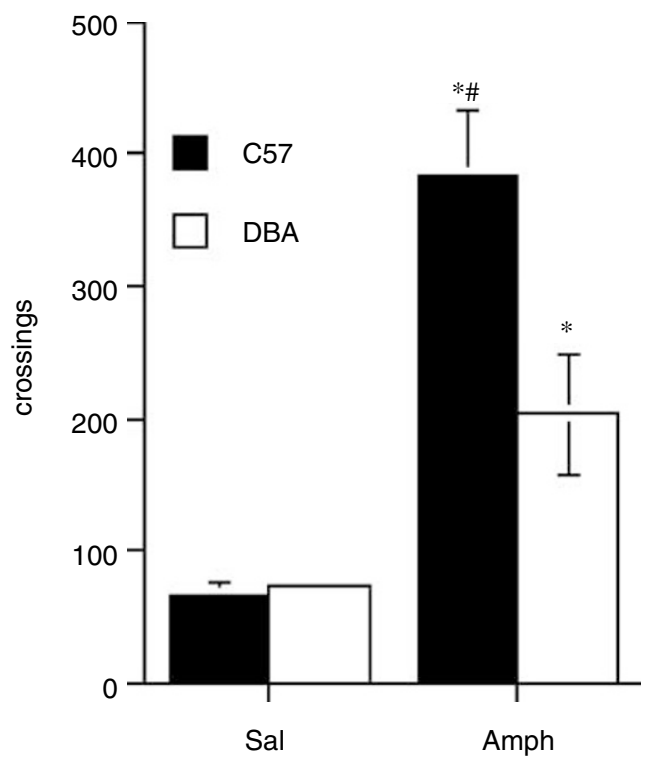

Figure 4 Effects of amphetamine ( $2.5 \mathrm{mg} / \mathrm{kg}$, i.p.) (Amph) or saline (Sal) on locomotor activity (mean crossings \pm SE) of naive C57BL/6) (C57) and DBA/2J (DBA) mice. ${ }^{*} p<0.01$ in comparison with saline (Sal); $\# p<0.0$ l in comparison with the other strain.

Table I DA and NE Tissue Levels (ng/g Wet Weight) in mpFC of Sham and DA-depleted DBA/2J Mice

\begin{tabular}{lrr}
\hline & NE & \multicolumn{1}{c}{ DA } \\
\hline Sham & $790 \pm 28$ & $191 \pm 17$ \\
DA depleted & $739 \pm 49$ & $53 \pm 8^{*}$ \\
\hline
\end{tabular}

${ }^{*} p<0.005$ in comparison with Sham.

A significantly higher DA outflow increase was evident in DA-depleted mice compared with Sham between 20 and 80 min after injection. Extracellular DA reached a $650 \%$ maximal increase (at $20 \mathrm{~min}$ ) in DA-depleted animals, while a $160 \%$ maximal increase was evident (at $40 \mathrm{~min}$ ) in the Sham group. No significant differences were evident in basal levels between groups (Figure 5).

The effects of selective DA depletion in the mpFC on locomotor activity are shown in Figure 6. Statistical analyses revealed a significant pretreatment $\times$ treatment interaction $(\mathrm{F}(1,27)=7.2 ; p<0.02)$. Individual comparisons showed that amphetamine increased locomotor activity in both groups, but DA-depleted animals exhibited dramatically higher locomotor activity scores than Sham animals. No significant differences were evident between DA-depleted and Sham animals injected with saline.

Effects of Amphetamine Applied to mpFC or to NAc on Dialysate DA Levels

Amphetamine infused through the microdialysis probe produced a clear-cut increase of extracellular DA in both the $\mathrm{mpFC} \quad(\mathrm{F}(5,24)=7.67 ; p<0.0005)$ and the NAc $(\mathrm{F}(5,30)=92.2 ; p<0.0005)$. In particular, the psychostimulant produced a dose-related parallel percent increase from the basal levels in the two brain areas, thus indicating a

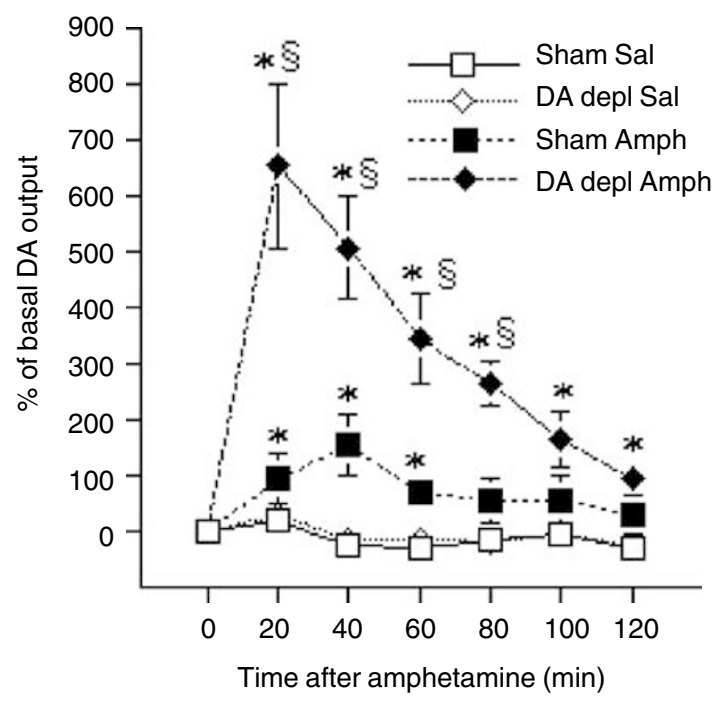

Figure 5 Effects of mpFC dopamine depletion (DA depl) on extracellular dopamine in the NAc of animals $(n=10-12$ per group) receiving saline (Sal) or amphetamine $(2.5 \mathrm{mg} / \mathrm{kg}$, i.p.) (Amph). Results are expressed as percent changes from basal levels. Statistical analyses were carried out on raw data (mean $\pm \mathrm{SE}$ ). $* p<0.05$ compared with saline; $\S p<0.05$ compared with the Sham group.

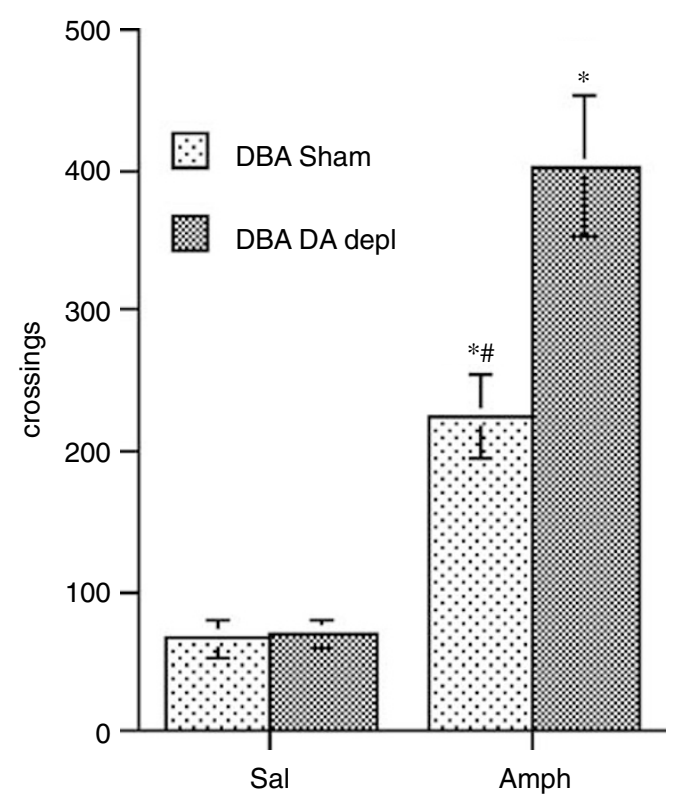

Figure 6 Effects of amphetamine ( $2.5 \mathrm{mg} / \mathrm{kg}$, i.p.) (Amph) or saline (Sal) on locomotor activity (mean crossings \pm SE) of DBA/2J (DBA) mice either bearing a selective DA depletion in the mpFC (DA depl) or sham lesioned (Sham). $* p<0.01$ in comparison with saline (Sal); $\# p<0.01$ in comparison with DA depl.

similar effect of local infusion in the mpFC and the NAc (Figure 7).

\section{DISCUSSION}

The present results show that mesocortical DA controls the genotype-dependent effects of systemic amphetamine on mesoaccumbems DA release and on locomotion. 
Since dopamine exerts a tonic stimulatory control on

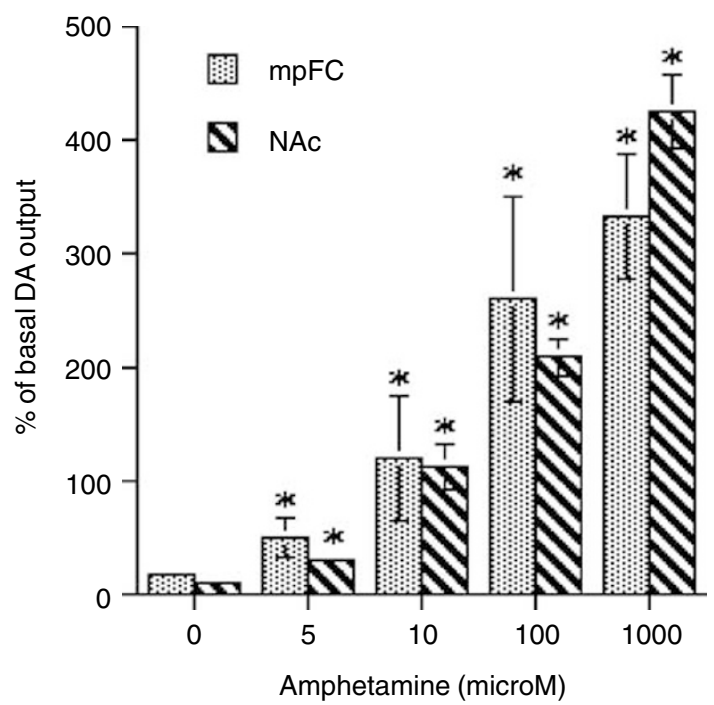

Figure 7 Effects of local amphetamine infusion (I, 5, 10, 100, 1000 $\mu \mathrm{M})$ on extracellular dopamine in the $\mathrm{mpFC}$ and the NAc of DBA/2J mice. Results are expressed as percent changes from basal levels. Statistical analyses were carried out on raw data. All data are mean \pm SE $(n=6$ per group). ${ }^{*} p<0.0$ I in comparison with basal values.

First, in response to systemic amphetamine challenge, DBA mice show higher mesocortical DA activation accompanied by a weak dopaminergic accumbal response, while the opposite occurs in the C57 background, a finding that supports the hypothesis of an inverse relationship between mesocortical and mesoaccumbens DA functioning. Indeed, we observed dramatic differences in the effects of systemic amphetamine on DA outflow in the mpFC of the two backgrounds. In fact, at all time points, DBA mice showed significantly higher DA responses than C57 mice. The effect of amphetamine on DA outflow lasted up to $120 \mathrm{~min}$ in DBA mice, while in C57 mice DA levels were no different from saline at $100 \mathrm{~min}$ postinjection. Moreover, amphetamine produced $\sim 700 \%$ maximal increase in DA release in the mpFC of DBA mice and $\sim 150 \%$ maximal increase in DA in the C57 strain.

Instead, amphetamine produced a higher DA release in the NAc of C57 strain than DBA mice, confirming previous results (Zocchi et al, 1998). In fact, the C57 strain showed $\sim 350 \%$ increase, while in DBA mice $\sim 150 \%$ maximal outflow occurred. It should be pointed out that DBA mice showed moderately higher DA basal levels in the mpFC than C57 $(+24 \%)$ that were conceivably too weak to lead to parallel lower DA basal levels in the NAc.

Consistent with the effects produced in the NAc, systemic amphetamine challenge induced a stronger increase of locomotor activity in C57 mice than the DBA background, in accordance with the consolidated literature (see for review Puglisi-Allegra and Cabib, 1997).

Second, prefrontal cortical DA depletion in DBA mice produced a dramatic increase in the accumbal DA response to the psychostimulant in comparison with the Sham group. These results are in agreement with the view that increased DA release in the mpFC has an inhibitory effect on accumbal DA release (Deutch et al, 1990; Le Moal and Simon, 1991; Piazza et al, 1991; Thompson and Moss, 1995; Doherty and Gratton, 1996; Karreman and Moghaddam, 1996; Harden et al, 1998; Jentsch et al, 1998).
GABA interneurons in the prefrontal cortex (Penit-Soria et al, 1987; Pirot et al, 1992), it may be that 6-OHDAinduced DA depletion decreases the activity of GABA interneurons. This reduction could, in turn, trigger an increased activity of efferents to the NAc by removing the inhibition of pyramidal cells (Bunney and Aghajanian, 1976; Ferron et al, 1984).

To our knowledge, the present results provide the first demonstration of potentiation accumbal extracellular DA induced by acute amphetamine challenge by prefrontal DA depletion in rodents.

In addition, selective DA depletion in the mpFC leads to increased effects of amphetamine challenge on locomotor activity in DBA mice, which parallels the increased DA response in the NAc. The behavioral effects of prefrontal cortical DA depletion are in agreement with previous results obtained in rat (Pycock et al, 1980; Bjijou et al, 2002), but not with others (King et al, 1997). It is worth noting that selective DA depletion of $\mathrm{pFC}$ has been shown to increase the effects of systemic cocaine on DA outflow in the NAc as well as on locomotion, thus leading to sensitization-like effects in rats receiving acute psychostimulant challenge (Beyer and Steketee, 1999).

Third, the results from reverse microdialysis experiments support the fact that the low DA activation in the NAc of the DBA background following systemic amphetamine depends on an inhibitory action outside the accumbens. Indeed, the local infusion of amphetamine produces a comparable increase in extracellular DA in both the $\mathrm{mpFC}$ and the NAc, indicating that the effects of amphetamine on nerve terminals in the two brain areas are similar in potency. Therefore, the different responses by $\mathrm{mpFC}$ and NAc to systemic drug administration cannot be ascribed to differences in transporter-related mechanisms in the two brain areas (Moron et al, 2002), but involve an active inhibitory mechanism. In the light of the results obtained by the experiment on mesocortical DA depletion described above, this inhibitory role can be ascribed to prefrontal cortical DA transmission.

Our present results add new evidence, indicating a major role of prefrontal cortical DA transmission in the effects of psychostimulants on mesoaccumbens DA release and on behavioral output. Moreover, they indicate that individual differences in susceptibility to the addictive properties of psychostimulants depend on genotype-controlled balance between drug-induced mesocortical and mesoaccumbens DA response.

In fact, mice of the C57 strain that respond with higher mesoaccumbens dopamine release and lower prefrontal DA release to amphetamine challenge than mice of the DBA background are also more responsive to the stimulating effects of amphetamine than DBA mice. In the C57 background, a dramatically less pronounced effect of the psychostimulant on DA release in the $\mathrm{mpFC}$ is likely to produce a low inhibitory action on DA outflow in the NAc and, therefore, a more pronounced accumbal DA release and behavioral effects of amphetamine. Consistent with these findings, selective DA depletion in the mpFC of the DBA background makes these animals similar to C57 in terms of the effects of amphetamine on both accumbal DA release and behavioral output. 
In conclusion, the present results indicate a genetic control over the balance between mesocortical and mesoaccumbens DA response to amphetamine that determines the behavioral outcome following challenge with the psychostimulant.

These data have a greater clinical relevance, since imbalance between mesocortical and mesolimbic DA system has been proposed as a major etiological factor in psychoses (Deutch et al, 1990; Karreman and Moghaddam, 1996; Egan and Weinberger, 1997; Di Chiara et al, 1999) and in drug addiction (Piazza and Le Moal, 1996).

\section{ACKNOWLEDGEMENTS}

We thank Dr E Catalfamo and Dr C Castellano (CNR) for guidance in implantation and lesion experiments. This research has been supported by Ministero della Ricerca Scientifica e Tecnologica (COFIN 2001), Università 'La Sapienza' Ateneo (1999-2001), Ministero della Salute (Progetto Finalizzato RF00.96P 2001-2003).

\section{REFERENCES}

Beyer CE, Steketee JD (1999). Dopamine depletion in the medial prefrontal cortex induces sensitized-like behavioral and neurochemical responses to cocaine. Brain Res 833: 133-141.

Bjijou Y, De Deurwaerdere P, Spampinato U, Stinus L, Cador M (2002). D-Amphetamine-induced behavioral sensitization: effect of lesioning dopaminergic terminals in the medial prefrontal cortex, the amygdala and the entorhinal cortex. Neuroscience 109: 499-516.

Bunney BS, Aghajanian GK (1976). Dopamine and norepinephrine innervated cells in the rat prefrontal cortex: pharmacological differentiation using microiontophoretic techniques. Life Sci 19: 1783-1792.

Cabib S, Orsini C, Le Moal M, Piazza PV (2000). Abolition and reversal of strain differences in behavioral responses to drugs of abuse after a brief experience. Science 289: 463-465.

Deutch AY, Clark WA, Roth RH (1990). Prefrontal cortical dopamine depletion enhances the responsiveness of mesolimbic dopamine neurons to stress. Brain Res 521: 311-315.

Di Chiara G, Loddo P, Tanda G (1999). Reciprocal changes in prefrontal and limbic dopamine responsiveness to aversive and rewarding stimuli after chronic mild stress: implications for the psychobiology of depression. Biol Psychiatry 46: 1624-1633.

Doherty MD, Gratton A (1996). Medial prefrontal cortical D1 receptor modulation of the meso-accumbens dopamine response to stress: an electrochemical study in freely behaving rats. Brain Res 715: 86-97.

Egan MF, Weinberger DR (1997). Neurobiology of schizophrenia. Curr Opin Neurobiol 7: 701-707.

Ferron A, Thierry AM, Le Douarin C, Glowinski J (1984). Inhibitory influence of the mesocortical dopaminergic system on the spontaneous activity or excitatory response induced from the thalamic mediodorsal nucleus in the rat medial prefrontal cortex. Brain Res 302: 257-265.

Franklin KBJ, Paxinos G (1998). The Mouse Brain in Stereotaxic Coordinates. Academic Press: San Diego.

Harden DG, King D, Finlay JM, Grace AA (1998). Depletion of dopamine in the prefrontal cortex decreases the basal electrophysiological activity of mesolimbic dopamine neurons. Brain Res 794: 96-102.

Hedou G, Homberg J, Martin S, Wirth K, Reldon J, Heidbreder CA (2000). Effect of amphetamine on extracellular acetylcholine and monoamine levels in subterritories of the rat medial prefrontal cortex. Eur J pharmacol 390: 127-136.

Herve D, Simon H, Blanc G, Le Moal M, Glowinski J, Tassin JP (1981). Opposite changes in dopamine utilization in the nucleus accumbens and the frontal cortex after electrolytic lesion of the median raphe in the rat. Brain Res 216: 422-428.

Jackson ME, Moghaddam B (2001). Amygdala regulation of nucleus accumbens dopamine output is governed by the prefrontal cortex. J Neurosci 21: 676-681.

Jentsch JD, Tran A, Taylor JR, Roth RH (1998). Prefrontal cortical involvement in phencyclidine-induced activation of the mesolimbic system: behavioral and neurochemical evidence. Psychopharmacology 138: 89-95.

Jones SR, O'Dell SJ, Marshall JF, Wightman RM (1996). Functional and anatomical evidence for different dopamine dynamics in the core and shell of the nucleus accumbens in slices of rat brain. Synapse 23: 224-231.

Karreman M, Moghaddam B (1996). The prefrontal cortex regulates the basal release of dopamine in the limbic striatum: an effect mediated by ventral tegmental area. J Neurochem 66: 589-598.

King D, Zigmond MJ, Finlay JM (1997). Effects of dopamine depletion in the medial prefrontal cortex on the stress-induced increase in extracellular dopamine in the nucleus accumbens core and shell. Neuroscience 77: 141-153.

Le Moal M, Simon H (1991). Mesocorticolimbic dopaminergic network: functional and regulatory role. Physiol Rev 71: 155-234.

Moron JA, Brockington A, Wise R, Rocha BA, Hope BT (2002). Dopamine uptake through the norepinephrine transporter in brain regions with low levels of the dopamine transporter: evidence from knock-out mouse lines. J Neurosci 22: 389-395.

Nirenberg MJ, Chan J, Pohorille A, Vaughan RA, Uhl GR, Kuhar MJ et al (1997). The dopamine transporter: comparative ultrastructure of dopaminergic axons in limbic and motor compartments of the nucleus accumbens. J Neurosci 17: 6899-6907.

Penit-Soria J, Audinat E, Crepel F (1987). Excitation of rat prefrontal cortical neurons by dopamine: an in vivo electrophysiological study. Brain Res 425: 363-374.

Piazza PV, Le Moal M (1996). Pathophysiological basis of vulnerability to drug abuse: role of an interaction between stress, glucocorticoids and dopaminergic neurons. Rev Pharmacol Toxicol 36: 359-378.

Piazza PV, Rouge-Pont F, Deminiere JM, Kharoubi M, Le Moal M, Simon H (1991). Dopaminergic activity is reduced in the prefrontal cortex and increased in the nucleus accumbens of rats predisposed to develop amphetamine self-administration. Brain Res 567: 169-174.

Pirot S, Godbout R, Mantz J, Tassin JP, Glowinski J, Thierry AM (1992). Inhibitory effect of ventral tegmental area stimulation on the activity of prefrontal cortical neurons: evidence for the involvement of both dopaminergic and GABAergic components. Neuroscience 49: 857-865.

Porrino LJ, Lyons D (2000). Orbital and medial prefrontal cortex and psychostimulant abuse: studies in animal models. Cereb Cortex 10: 326-333.

Puglisi-Allegra S, Cabib S (1997). Psychopharmacology of dopamine: the contribution of comparative studies in inbred strains of mice. Prog Neurobiol 51: 637-661.

Pycock CY, Carter CJ, Kervin RW (1980). Effects of 6-hydroxydopamine lesions of the medial prefrontal cortex on neurotransmitter systems in subcortical sites in the rat. $J$ Neurochem 34: 91-99.

Spink AJ, Tegelenbosch RA, Buma MO, Noldus LP (2001). The EthoVision video tracking system - a tool for behavioral phenotyping of transgenic mice. Physiol Behav 73: 731-744. 
Thompson TL, Moss RL (1995). In vivo stimulated dopamine release in the nucleus accumbens: modulation by the prefrontal cortex. Brain Res 686: 93-98.

Westerink BHC, Enrico P, Feimann J, De Vries JB (1998). The pharmacology of mesocortical dopamine neurons. A dual-probe microdialysis study in the ventral tegmental area and prefrontal cortex of the rat brain. J Pharmacol Exp Ther 285: 143-154.

Ventura R, Cabib S, Alcaro A, Orsini C, Puglisi-Allegra S (2003). Norepinephrine in the prefrontal cortex is critical for amphe- tamine-induced reward and mesoaccumbens dopamine release. $J$ Neurosci 23: 1879-1885.

Ventura R, Cabib S, Puglisi-Allegra S (2001). Opposite genotypedependent mesocorticolimbic dopamine response to stress. Neuroscience 104: 627-631.

Zocchi A, Orsini C, Cabib S, Puglisi-Allegra S (1998). Parallel strain-dependent effect of amphetamine on locomotor acivity and dopamine release in the nucleus accumbens: an in vivo study in mice. Neuroscience 82: 521-528. 